
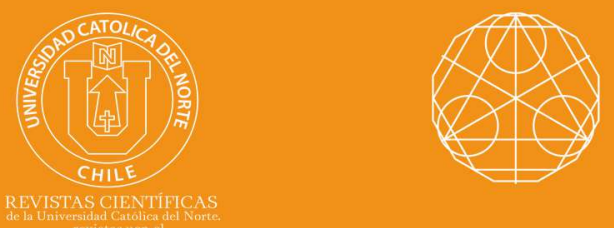

\title{
Some trapezoid and midpoint type inequalities for newly defined quantum integrals
}

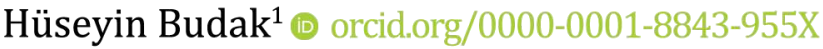

${ }^{1}$ Düzce University, Dept. of Mathematics, Faculty of Science and Arts, Düzce, Turkey. ఐhsyn.budak@gmail.com

\section{Abstract:}

In this paper, we first obtain prove two new identities for the quantum integrals. Then we establish Trapezoid and Midpoint type inequalities for quantum integrals defined by Bermudo et al. in [3]. The inequalities in this study generalize some results obtained in earlier works

Keywords: Hermite-Hadamard inequality; q-integral; Quantum calculus; Convex function; Trapezoid; Midpoint.

MSC (2020): 34A08, 26A51, 26D15.

\section{Cite this article as (IEEE citation style): \\ H. Budak, "Some trapezoid and midpoint type inequalities for newly defined quantum integrals", Proyecciones (Antofagasta, On line), vol. 40, no. 1, pp. 199-215, 2021 doi: 10.22199/issn.0717-6279-2021-01-0013}

Article copyright: (C) 2021 Hüseyin Budak. This is an open access article distributed under the terms of the Creative Commons License, which permits unrestricted use and distribution provided the original author and source are credited. 


\section{Introduction}

The Hermite-Hadamard inequality discovered by C. Hermite and J. Hadamard (see, e.g., [4], [14, p.137]) is one of the most well established inequalities in the theory of convex functions with a geometrical interpretation and many applications. These inequalities state that if $f: I \rightarrow \mathbf{R}$ is a convex function on the interval $I$ of real numbers and $a, b \in I$ with $a<b$, then

$$
f\left(\frac{\bar{a}+\underline{b}}{2}\right) \leq \frac{1}{b-a} \int_{a}^{b} f(x) d x \leq \frac{f(a)+f(b)}{2} .
$$

Both inequalities hold in the reversed direction if $f$ is concave. We note that Hermite-Hadamard inequality may be regarded as a refinement of the concept of convexity and it follows easily from Jensen's inequality. HermiteHadamard inequality for convex functions has received renewed attention in recent years and a remarkable variety of refinements and generalizations have been studied.

The general structure of this paper consist of five main sections including introduction. In Section 2, we give some necessary important notations for concept $q$-calculus and we also mention some related works in the literature. In section 3 and Section 4, we provide Trapezoid and Midpoint type inequalities for $q^{b}$ integrals, respectively. We also examine the relation between our results and inequalities presented in the earlier works. Finally, in Section 5, some conclusions and further directions of research are discussed. We note that the opinion and technique of this work may inspire new research in this area.

\section{Preliminaries of $q$-Calculus and Some Inequalities}

Many integral inequalities well known in classical analysis such as Hölder inequality, Hermite-Hadamard inequality and Ostrowski inequality, CauchyBunyakovsky-Schwarz, Gruss, Gruss- Cebysev and other integral inequalities have been proved and applied for $q$-calculus using classical convexity.For the other results for $q$-calculus please refer to $[1,2,7,6,12,13,15,17]$. In this section we present some required definitions and related inequalities about $q$-calculus. Also, here and further we use the following notation(see $[9])$ :

$$
[n]_{q}=\frac{1-q^{n}}{1-q}=1+q+q^{2}+\ldots+q^{n-1}, \quad q \in(0,1) .
$$


In [8], Jackson gave the $q$-Jackson integral from 0 to $b$ for $0<q<1$ asfollows:

$$
\int_{0}^{b} f(x) \quad d_{q} x=(1-q) b \sum_{n=0}^{\infty} q^{n} f\left(b q^{n}\right)
$$

provided the sum converge absolutely.

Jackson in [8] gave the $q$-Jackson integral in a generic interval $[a, b]$ as:

$$
\int_{a}^{b} f(x) d_{q} x=\int_{0}^{b} f(x) d_{q} x-\int_{0}^{a} f(x) d_{q} x .
$$

Definition 1. [16] For a continuous function $f:[a, b] \rightarrow \mathbf{R}$, then $q_{a^{-}}$ derivative of $f$ at $x \in[a, b]$ is characterized by the expression

$$
{ }_{a} D_{q} f(x)=\frac{f(x)-f(q x+(1-q) a)}{(1-q)(x-a)}, x=a .
$$

Since $f:[a, b] \rightarrow \mathbf{R}$ is a continuous function, thus we have

$$
{ }_{a} D_{q} f(a)=x \rightarrow a \lim _{a} D_{q} f(x) .
$$

The function $f$ is said to be $q$ - differentiable on $[a, b]$ if

$$
{ }_{a} D_{q} f(t)
$$

exists for all $x \in[a, b]$. If $a=0$ in (2.2), then ${ }_{0} D_{q} f(x)=D_{q} f(x)$

where

$$
D_{q} f(x)
$$

is familiar $q$-derivative of $f$ at $x \in[a, b]$ defined by the expression (see [9])

$$
D_{q} f(x)=\frac{f(x)-f(q x)}{(1-q) x}, x=0 .
$$

Definition 2. [3] For a continuous function $f:[a, b] \rightarrow \mathbf{R}$, then $q^{b}$-derivative of $f$ at $x \in[a, b]$ is characterized by the expression

$$
{ }^{b} D_{q} f(x)=\frac{f(q x+(1-q) b)-f(x)}{(1-q)(b-x)}, x=b .
$$


Definition 3. [16] Let $f:[a, b] \rightarrow \mathbf{R}$ be a continuous function. Then, the $q_{a}$-definite integral on $[a, b]$ is defined as

$$
\begin{aligned}
\int_{a}^{b} f(x)_{a} d_{q} x & =(1-q)(b-a) \sum_{n=0}^{\infty} q^{n} f\left(q^{n} b+\left(1-q^{n}\right) a\right) \\
& =(b-a) \int_{0}^{1} f((1-t) a+t b) d_{q} t .
\end{aligned}
$$

In [1], Alp et al. proved the following $q_{a}$-Hermite-Hadamard inequalities for convex functions in the setting of quantum calculus:

Theorem 1. Let $f:[a, b] \rightarrow \mathbf{R}$ be a convex differentiable function on $[a, b]$ and $0<q<1$. Then $q$-Hermite-Hadamard inequalities are as follows:

$$
f\left(\frac{\bar{q} \underline{a} \pm \underline{b}}{1+q}\right) \leq \frac{1}{b-a} \int_{a}^{b} f(x){ }_{a} d_{q} x \leq \frac{q f(a)+f(b)}{1+q} .
$$

In [11] and [1], authors established some bounds for left and right hand sides of the inequality (2.3).

On the other hand, Bermudo et al. gave the following new definition and related Hermite-Hadamard type inequalities:

Definition 4. [3] Let $f:[a, b] \rightarrow \mathbf{R}$ be a continuous function. Then, the $q^{b}$-definite integral on $[a, b]$ is defined as

$$
\begin{aligned}
\int_{a}^{b} f(x)^{b} d_{q} x & =(1-q)(b-a) \sum_{n=0}^{\infty} q^{n} f\left(q^{n} a+\left(1-q^{n}\right) b\right) \\
& =(b-a) \int_{0}^{1} f(t a+(1-t) b) d_{q} t .
\end{aligned}
$$

Theorem 2. [3] Let $f:[a, b] \rightarrow \mathbf{R}$ be a convex function on $[a, b]$ and $0<q<1$. Then, $q$-Hermite-Hadamard inequalities are as follows:

$$
f\left(\frac{a+q b}{1+q}\right) \leq \frac{1}{b-a} \int_{a}^{b} f(x){ }^{b} d_{q} x \leq \frac{f(a)+q f(b)}{1+q} .
$$


From Theorem 1 and Theorem 2, one can the following inequalities:

Corollary 1. [3] For any convex function $f:[a, b] \rightarrow \mathbf{R}$ and $0<q<1$, we have

$f\left(\frac{q a+b}{1+q}\right)+f\left(\frac{a+q b}{1+q}\right) \leq \frac{1}{b-a}\left\{\int_{a}^{b} f(x){ }_{a} d_{q} x+\int_{a}^{b} f(x)^{b} d_{q} x\right\} \leq f(a)+f(b)$

and

(2.6) $f\left(\frac{a+b}{2}\right) \leq \frac{1}{2(b-a)}\left\{\int_{a}^{b} f(x)_{a} d_{q} x+\int_{a}^{b} f(x)^{b} d_{q} x\right\} \leq \frac{f(a)+f(b)}{2}$.

In this paper we will find some bounds for the left and right hand sides of the inequality (2.4).

\section{New Trapezoid Type Inequalities for Quantum Integrals}

In this section we will prove some new Trapezoid type inequalities for functions whose $q^{b}$-derivatives are convex.

Lemma 1. Let $f:[a, b] \subset \mathbf{R} \rightarrow \mathbf{R}$ be a q-differentiable function on $(a, b)$ with ${ }^{b} D_{q} f$ be continuous and integrable on $[a, b]$, then

$$
\begin{gathered}
\frac{f(a)+q f(b)}{1+q}-\frac{1}{b-a} \int_{a}^{b} f(x)^{b} d_{q} x \\
=\frac{q(b-a)}{1+q} \int_{0}^{1}(1-(1+q) t)^{b} D_{q} f(t a+(1-t) b) d_{q} t
\end{gathered}
$$

where $0<q<1$

Proof. By the Definition 2, we have

$$
{ }^{b} D_{q} f(t a+(1-t) b)=\frac{f(q t a+(1-q t) b)-f(t a+(1-t) b)}{(1-q)(b-a) t} .
$$


Then it follows that

$$
\begin{gathered}
\int_{0}^{1}(1-(1+q) t)^{b} D_{q} f(t a+(1-t) b) d_{q} t \\
=\int_{0}^{1}(1-(1+q) t) \frac{f(q t a+(1-q t) b)-f(t a+(1-t) b)}{(1-q)(b-a) t} d_{q} t \\
=\frac{1}{b-a} \int_{0}^{1} \frac{f(q t a+(1-q t) b)-f(t a+(1-t) b)}{(1-q) t} d_{q} t \\
-\frac{1+q}{b-a} \int_{0}^{1} \frac{f(q t a+(1-q t) b)-f(t a+(1-t) b)}{(1-q)} d_{q} t .
\end{gathered}
$$

By the equality (2.1), we have

$$
\begin{gathered}
\frac{1}{b-a} \int_{0}^{1} \frac{f(q t a+(1-q t) b)-f(t a+(1-t) b)}{(1-q) t} d_{q} t \\
=\frac{1}{b-a} \sum_{k=0}^{\infty} f\left(q^{k+1} a+\left(1-q^{k+1}\right) b\right)-\frac{1}{b-a} \sum_{k=0}^{\infty} f\left(q^{k} a+\left(1-q^{k}\right) b\right) \\
=\frac{1}{b-a}[f(b)-f(a)] .
\end{gathered}
$$

By the equality (2.1) and Definition 4, we get

$$
\begin{gathered}
\frac{1+q}{b-a} \int_{0}^{1} \frac{f(q t a+(1-q t) b)-f(t a+(1-t) b)}{(1-q)} d_{q} t \\
=\frac{1+q}{b-a} \sum_{k=0}^{\infty} q^{k} f\left(q^{k+1} a+\left(1-q^{k+1}\right) b\right)-\frac{1+q}{b-a} \sum_{k=0}^{\infty} q^{k} f\left(q^{k} a+\left(1-q^{k}\right) b\right) \\
=\frac{1+q}{q(b-a)} \sum_{k=1}^{\infty} q^{k} f\left(q^{k} a+\left(1-q^{k}\right) b\right)-\frac{1+q}{b-a} \sum_{k=0}^{\infty} q^{k} f\left(q^{k} a+\left(1-q^{k}\right) b\right)
\end{gathered}
$$




$$
\begin{gathered}
=\frac{(1+q)(1-q)}{q(b-a)} \sum_{k=0}^{\infty} q^{k} f\left(q^{k} a+\left(1-q^{k}\right) b\right)-\frac{1+q}{q(b-a)} f(a) \\
=\frac{(1+q)}{q(b-a)^{2}} \int_{a}^{b} f(x)^{b} d_{q} x-\frac{1+q}{q(b-a)} f(a) .
\end{gathered}
$$

By substitute the equalities (3) and (3) in (3), we have

$$
\begin{gathered}
\int_{0}^{1}(1-(1+q) t)^{b} D_{q} f(t a+(1-t) b) d_{q} t \\
=-\frac{(1+q)}{q(b-a)^{2}} \int_{a}^{b} f(x)^{b} d_{q} x+\frac{1+q}{q(b-a)} f(a)+\frac{1}{b-a}[f(b)-f(a)] \\
=\frac{(1+q)}{q(b-a)}\left[\frac{f(a)+q f(b)}{1+q}-\frac{1}{b-a} \int_{a}^{b} f(x)^{b} d_{q} x\right]
\end{gathered}
$$

which completes the proof.

Remark 1. If we take the limit $q \rightarrow 1^{-}$in Lemma 1, then Lemma 1 reduces to [5, Lemma 2.1].

Theorem 1. Let $f:[a, b] \subset \mathbf{R} \rightarrow \mathbf{R}$ be a $q$-differentiable function on $(a, b)$ with ${ }^{b} D_{q} f$ be continuous and integrable on $[a, b]$. If $\left|{ }^{b} D_{q} f\right|$ is convex on $[a, b]$ then we have the inequality

$$
\begin{aligned}
& \left|\frac{f(a)+q f(b)}{1+q}-\frac{1}{b-a} \int_{a}^{b} f(x){ }^{b} d_{q} x\right| \\
\leq & (b-a)\left[\left|{ }^{b} D_{q} f(a)\right| \frac{q^{2}\left(1+4 q+q^{2}\right)}{\left(1+q+q^{2}\right)(1+q)^{4}}+\left|{ }^{b} D_{q} f(b)\right| \frac{q^{2}\left(1+3 q^{2}+2 q^{3}\right)}{\left(1+q+q^{2}\right)(1+q)^{4}}\right]
\end{aligned}
$$

where $0<q<1$. 
Proof. Taking modulus in Lemma 1 and using convexity of $\left|b^{b} D_{q} f\right|$, we obtain

$$
\begin{gathered}
\quad\left|\frac{f(a)+q f(b)}{1+q}-\frac{1}{b-a} \int_{a}^{b} f(x)^{b} d_{q} x\right| \\
=\frac{q(b-a)}{1+q}\left|\int_{0}^{1}(1-(1+q) t)^{b} D_{q} f(t a+(1-t) b) d_{q} t\right| \\
\leq \frac{q(b-a)}{1+q} \int_{0}^{1}|(1-(1+q) t)|{ }^{b} D_{q} f(t a+(1-t) b) \mid d_{q} t \\
\leq \frac{q(b-a)}{1+q} \int_{0}^{1}|(1-(1+q) t)|\left[t\left|{ }^{b} D_{q} f(a)\right|+(1-t)\left|{ }^{b} D_{q} f(b)\right|\right] d_{q} t \\
=\frac{q(b-a)}{1+q}\left[\left|{ }^{b} D_{q} f(a)\right| \int_{0}^{1}|(1-(1+q) t)| t d_{q} t+\left|{ }^{b} D_{q} f(b)\right| \int_{0}^{1}|(1-(1+q) t)|(1-t) d_{q} t\right] \\
=\frac{q(b-a)}{1+q}\left[\left|{ }^{b} D_{q} f(a)\right| \frac{q\left(1+4 q+q^{2}\right)}{\left(1+q+q^{2}\right)(1+q)^{3}}+\left|{ }^{b} D_{q} f(b)\right| \frac{q\left(1+3 q^{2}+2 q^{3}\right)}{\left(1+q+q^{2}\right)(1+q)^{3}}\right]
\end{gathered}
$$

which completes the proof.

Remark 2. If we take the limit $q \rightarrow 1^{-}$in Theorem 1 , then Theorem 1 reduces to [5, Theorem 2.2].

Theorem 2. Let $f:[a, b] \subset \mathbf{R} \rightarrow \mathbf{R}$ be a $q$-differentiable function on $(a, b)$ with ${ }^{b} D_{q} f$ be continuous and integrable on $[a, b]$. If $\left|{ }^{b} D_{q} f\right|^{p_{1}}, p_{1} \geq 1$, is convex on $[a, b]$ then we have the inequality

$$
\begin{aligned}
& \left|\frac{f(a)+q f(b)}{1+q}-\frac{1}{b-a} \int_{a}^{b} f(x){ }^{b} d_{q} x\right| \\
\leq & \frac{q(b-a)}{1+q}\left(\frac{q\left(2+q+q^{3}\right)}{(1+q)^{3}}\right)^{1-\frac{1}{p_{1}}} \\
& \times\left(\frac{q\left(1+4 q+q^{2}\right)}{\left(1+q+q^{2}\right)(1+q)^{3}}\left|{ }^{b} D_{q} f(a)\right|^{p_{1}}+\frac{q^{2}\left(1+3 q^{2}+2 q^{3}\right)}{\left(1+q+q^{2}\right)(1+q)^{3}}\left|{ }^{b} D_{q} f(b)\right|^{p_{1}}\right)^{\frac{1}{p_{1}}}
\end{aligned}
$$

where $0<q<1$. 
Proof. Taking modulus in Lemma 1 and using the power mean inequality, we have

$$
\begin{gathered}
\left|\frac{f(a)+q f(b)}{1+q}-\frac{1}{b-a} \int_{a}^{b} f(x)^{b} d_{q} x\right| \\
\leq \frac{q(b-a)}{1+q} \int_{0}^{1}|(1-(1+q) t)|\left|{ }^{b} D_{q} f(t a+(1-t) b)\right| d_{q} t \\
\leq \frac{q(b-a)}{1+q}\left(\int_{0}^{1}|(1-(1+q) t)| d_{q} t\right)^{1-\frac{1}{p_{1}}} \\
\left(\int_{0}^{1}|(1-(1+q) t)|\left|{ }^{b} D_{q} f(t a+(1-t) b)\right|^{p_{1}} d_{q} t\right)^{\frac{1}{p_{1}}} .
\end{gathered}
$$

Since $\left|{ }^{b} D_{q} f\right|^{p_{1}}$ is convex, we have

$$
\begin{aligned}
& \int_{0}^{1}|(1-(1+q) t)|\left|{ }^{b} D_{q} f(t a+(1-t) b)\right|^{p_{1}} d_{q} t \\
\leq & \int_{0}^{1}|(1-(1+q) t)|\left[t\left|{ }^{b} D_{q} f(a)\right|^{p_{1}}+(1-t)\left|{ }^{b} D_{q} f(b)\right|^{p_{1}}\right] d_{q} t \\
= & \frac{q\left(1+4 q+q^{2}\right)}{\left(1+q+q^{2}\right)(1+q)^{3}}\left|{ }^{b} D_{q} f(a)\right|^{p_{1}}+\frac{q^{2}\left(1+3 q^{2}+2 q^{3}\right)}{\left(1+q+q^{2}\right)(1+q)^{3}}\left|{ }^{b} D_{q} f(b)\right|^{p_{1}} .
\end{aligned}
$$

We also have

$$
\int_{0}^{1}|(1-(1+q) t)| d_{q} t=\frac{q\left(2+q+q^{3}\right)}{(1+q)^{3}} .
$$

This completes the proof.

\section{New Midpoint Type Inequalities for Quantum Integrals}

In this section we will prove some new Midpoint inequalities for functions whose $q^{b}$-derivatives are convex. 
Lemma 2. Let $f:[a, b] \subset \mathbf{R} \rightarrow \mathbf{R}$ be a $q$-differentiable function on $(a, b)$ with ${ }^{b} D_{q} f$ be continuous and integrable on $[a, b]$, then

$$
\begin{gathered}
q(b-a)\left[\int_{0}^{\frac{1}{1+q}} t^{b} D_{q} f(t a+(1-t) b) d_{q} t+\int_{\frac{1}{1+q}}^{1}\left(t-\frac{1}{q}\right)^{b} D_{q} f(t a+(1-t) b) d_{q} t\right] \\
=\frac{1}{b-a} \int_{a}^{b} f(x)^{b} d_{q} x-f\left(\frac{a+q b}{1+q}\right)
\end{gathered}
$$

where $0<q<1$.

Proof. We have

$$
\begin{gathered}
q(b-a)\left[\int_{0}^{\frac{1}{1+q}} t^{b} D_{q} f(t a+(1-t) b) d_{q} t+\int_{\frac{1}{1+q}}^{1}\left(t-\frac{1}{q}\right)^{b} D_{q} f(t a+(1-t) b) d_{q} t\right] \\
=q(b-a)\left[\int_{0}^{1} t^{b} D_{q} f(t a+(1-t) b) d_{q} t-\frac{1}{q} \int_{\frac{1}{1+q}}^{1} D_{q} f(t a+(1-t) b) d_{q} t\right] \\
=q(b-a)\left[\int_{0}^{1} t^{b} D_{q} f(t a+(1-t) b) d_{q} t-\frac{1}{q} \int_{0}^{1} D_{q} f(t a+(1-t) b) d_{q} t\right. \\
\left.+\frac{1}{q} \int_{0}^{\frac{1}{1+q}} b D_{q} f(t a+(1-t) b) d_{q} t\right] .
\end{gathered}
$$

By the equality (2.1), we have

$$
\begin{aligned}
& \int_{0}^{1} t^{b} D_{q} f(t a+(1-t) b) d_{q} t=\int_{0}^{1} \frac{f(q t a+(1-q t) b)-f(t a+(1-t) b)}{(1-q)(b-a)} d_{q} t \\
& =\frac{1}{b-a} \sum_{k=0}^{\infty} q^{k} f\left(q^{k+1} a+\left(1-q^{k+1}\right) b\right)-\frac{1}{b-a} \sum_{k=0}^{\infty} q^{k} f\left(q^{k} a+\left(1-q^{k}\right) b\right)
\end{aligned}
$$




$$
\begin{gathered}
=\frac{1}{q(b-a)} \sum_{k=1}^{\infty} q^{k} f\left(q^{k} a+\left(1-q^{k}\right) b\right)-\frac{1}{b-a} \sum_{k=0}^{\infty} q^{k} f\left(q^{k} a+\left(1-q^{k}\right) b\right) \\
=\frac{1}{b-a}\left(\frac{1}{q}-1\right) \sum_{k=1}^{\infty} q^{k} f\left(q^{k} a+\left(1-q^{k}\right) b\right)-\frac{f(a)}{q(b-a)} \\
=\frac{1}{q(b-a)} \int_{0}^{1} f(t a+(1-t) b) d_{q} t-\frac{f(a)}{q(b-a)} .
\end{gathered}
$$

Similarly we get

$$
\begin{gathered}
\frac{1}{q} \int_{0}^{1} b D_{q} f(t a+(1-t) b) d_{q} t=\frac{1}{q} \int_{0}^{1} \frac{f(q t a+(1-q t) b)-f(t a+(1-t) b)}{(1-q)(b-a) t} d_{q} t \\
=\frac{1}{q(b-a)} \sum_{k=0}^{\infty} f\left(q^{k+1} a+\left(1-q^{k+1}\right) b\right)-\frac{1}{q(b-a)} \sum_{k=0}^{\infty} f\left(q^{k} a+\left(1-q^{k}\right) b\right) \\
=\frac{1}{q(b-a)} \sum_{k=0}^{\infty}\left[f\left(q^{k+1} a+\left(1-q^{k+1}\right) b\right)-f\left(q^{k} a+\left(1-q^{k}\right) b\right)\right] \\
=\frac{1}{q(b-a)}[f(b)-f(a)]
\end{gathered}
$$

and

$$
\begin{gathered}
\frac{1}{q} \int_{0}^{\frac{1}{1+q}} b_{q} f(t a+(1-t) b) d_{q} t=\frac{1}{q} \int_{0}^{\frac{1}{1+q}} \frac{f(q t a+(1-q t) b)-f(t a+(1-t) b)}{(1-q)(b-a) t} d_{q} t \\
=\frac{1}{q(b-a)} \sum_{k=0}^{\infty} f\left(\frac{q^{k+1}}{1+q} a+\left(1-\frac{q^{k+1}}{1+q}\right) b\right)-\frac{1}{q(b-a)} \sum_{k=0}^{\infty} f\left(\frac{q^{k}}{1+q} a+\left(1-\frac{q^{k}}{1+q}\right) b\right) \\
=\frac{1}{q(b-a)} \sum_{k=0}^{\infty}\left[f\left(\frac{q^{k+1}}{1+q} a+\left(1-\frac{q^{k+1}}{1+q}\right) b\right)-f\left(\frac{q^{k}}{1+q} a+\left(1-\frac{q^{k}}{1+q}\right) b\right)\right] \\
=\frac{1}{q(b-a)}\left[-f\left(\frac{a+q b}{1+q}\right)-f(b)\right] .
\end{gathered}
$$


If we substitute the equalities (4)-(??) in (4), we get

$$
\begin{gathered}
q(b-a)\left[\int_{0}^{\frac{1}{1+q}} t^{b} D_{q} f(t a+(1-t) b) d_{q} t+\int_{\frac{1}{1+q}}^{1}\left(t-\frac{1}{q}\right)^{b} D_{q}\right. \\
f(t a+(1-t) b) d_{q} t \\
=\frac{1}{q(b-a)} \int_{0}^{1} f(t a+(1-t) b) d_{q} t-f\left(\frac{a+q b}{1+q}\right) \\
=\frac{1}{b-a} \int_{a}^{b} f(x)^{b} d_{q} x-f\left(\frac{a+q b}{1+q}\right)
\end{gathered}
$$

which completes the proof.

Remark 3. If we take the limit $q \rightarrow 1^{-}$in Lemma 2, then Lemma 2 reduces to [10, Lemma 2.1].

Theorem 1. Let $f:[a, b] \subset \mathbf{R} \rightarrow \mathbf{R}$ be a $q$-differentiable function on $(a, b)$ with ${ }^{b} D_{q} f$ be continuous and integrable on $[a, b]$. If $\left|{ }^{b} D_{q} f\right|$ is convex on $[a, b]$ then we have the inequality

$$
\left|\frac{1}{b-a} \int_{a}^{b} f(x)^{b} d_{q} x-f\left(\frac{a+q b}{1+q}\right)\right|
$$

$\leq q(b-a)\left[\left|{ }^{b} D_{q} f(a)\right| \frac{3}{(1+q)^{3}\left(1+q+q^{2}\right)}+\left|{ }^{b} D_{q} f(b)\right| \frac{-1+2 q+2 q^{2}}{(1+q)^{3}\left(1+q+q^{2}\right)}\right]$

where $0<q<1$.

Proof. Taking modulus in Lemma 2 and using convexity of $\left|{ }^{b} D_{q} f\right|$, we obtain

$$
\begin{gathered}
\left|\frac{1}{b-a} \int_{a}^{b} f(x)^{b} d_{q} x-f\left(\frac{a+q b}{1+q}\right)\right| \\
\leq q(b-a)\left[\left|\int_{0}^{\frac{1}{1+q}} t^{b} D_{q} f(t a+(1-t) b) d_{q} t\right|+\left|\int_{\frac{1}{1+q}}^{1}\left(t-\frac{1}{q}\right)^{b} D_{q} f(t a+(1-t) b) d_{q} t\right|\right]
\end{gathered}
$$




$$
\begin{aligned}
\leq q(b-a) & {\left[\int_{0}^{\frac{1}{1+q}} t\left|{ }^{b} D_{q} f(t a+(1-t) b)\right| d_{q} t+\int_{\frac{1}{1+q}}^{1}\left(\frac{1}{q}-t\right)\left|b D_{q} f(t a+(1-t) b)\right| d_{q} t\right] } \\
\leq & q(b-a)\left[\int_{0}^{\frac{1}{1+q}} t\left[\left.t\right|^{b} D_{q} f(a)|+(1-t)| b D_{q} f(b) \mid\right] d_{q} t\right. \\
& \left.+\int_{\frac{1}{1+q}}^{1}\left(\frac{1}{q}-t\right)\left[t\left|{ }^{b} D_{q} f(a)\right|+(1-t)\left|{ }^{b} D_{q} f(b)\right|\right] d_{q} t\right] \\
& =q(b-a)\left[\left|{ }^{b} D_{q} f(a)\right|\left(\int_{0}^{\frac{1}{1+q}} t^{2} d_{q} t+\int_{\frac{1}{1+q}}^{1}\left(\frac{1}{q}-t\right) t d_{q} t\right)\right. \\
+ & \left.\left|{ }^{b} D_{q} f(b)\right|\left[\int_{0}^{\frac{1}{1+q}} t(1-t) d_{q} t+\int_{\frac{1}{1+q}}^{1}\left(\frac{1}{q}-t\right)(1-t) d_{q} t\right)\right] .
\end{aligned}
$$

It can be easily that

$$
\begin{gathered}
\int_{0}^{\frac{1}{1+q}} t^{2} d_{q} t=\frac{1}{(1+q)^{3}\left(1+q+q^{2}\right)}, \\
\int_{\frac{1}{1+q}}^{1}\left(\frac{1}{q}-t\right) t=\frac{2}{(1+q)^{3}\left(1+q+q^{2}\right)}, \\
\int_{0}^{\frac{1}{1+q}} t(1-t) d_{q} t=\frac{q}{(1+q)^{2}\left(1+q+q^{2}\right)}
\end{gathered}
$$

and

$$
\int_{\frac{1}{1+q}}^{1}\left(\frac{1}{q}-t\right)(1-t) d_{q} t=\frac{-1+q+q^{2}}{(1+q)^{3}\left(1+q+q^{2}\right)} .
$$

By these equalities the proof is completed. 
Remark 4. If we take the limit $q \rightarrow 1^{-}$in Theorem 1 , then Theorem 1 reduces to [10, Theorem 2.2$]$.

Theorem 2. Let $f:[a, b] \subset \mathbf{R} \rightarrow \mathbf{R}$ be a $q$-differentiable function on $(a, b)$ with ${ }^{b} D_{q} f$ be continuous and integrable on $[a, b]$. If $\left|{ }^{b} D_{q} f\right|^{p_{1}}, p_{1} \geq 1$, is convex on $[a, b]$ then we have the inequality

$$
\begin{aligned}
& \left|\frac{1}{b-a} \int_{a}^{b} f(x)^{b} d_{q} x-f\left(\frac{a+q b}{1+q}\right)\right| \\
\leq & q(b-a)\left(\frac{1}{(1+q)^{3}}\right)^{1-\frac{1}{p_{1}}} \\
& \times\left[\left(\frac{1}{(1+q)^{3}\left(1+q+q^{2}\right)}\left|{ }^{b} D_{q} f(a)\right|^{p_{1}}+\frac{q}{(1+q)^{2}\left(1+q+q^{2}\right)}\left|{ }^{b} D_{q} f(b)\right|^{p_{1}}\right)^{\frac{1}{p_{1}}}\right. \\
& \left.\left(\frac{2}{(1+q)^{3}\left(1+q+q^{2}\right)}\left|{ }^{b} D_{q} f(a)\right|^{p_{1}}+\frac{-1+q+q^{2}}{(1+q)^{3}\left(1+q+q^{2}\right)}\left|{ }^{b} D_{q} f(b)\right|^{p_{1}}\right)^{\frac{1}{p_{1}}}\right]
\end{aligned}
$$

where $0<q<1$.

Proof. Taking modulus in Lemma 2 and using the power mean inequality, we obtain

$$
\begin{aligned}
& \left|\frac{1}{b-a} \int_{a}^{b} f(x){ }^{b} d_{q} x-f\left(\frac{a+q b}{1+q}\right)\right| \\
\leq & q(b-a)\left[\left(\int_{0}^{\frac{1}{1+q}} t d_{q} t\right)^{1-\frac{1}{p_{1}}}\left(\int_{0}^{\frac{1}{1+q}} t\left|{ }^{b} D_{q} f(t a+(1-t) b)\right|^{p_{1}} d_{q} t\right)^{\frac{1}{p_{1}}}\right. \\
& \left.\left(\int_{\frac{1}{1+q}}^{1}\left(\frac{1}{q}-t\right) d_{q} t\right)^{1-\frac{1}{p_{1}}}\left(\int_{\frac{1}{1+q}}^{1}\left(\frac{1}{q}-t\right)\left|{ }^{b} D_{q} f(t a+(1-t) b)\right|^{p_{1}} d_{q} t\right)^{\frac{1}{p_{1}}}\right] .
\end{aligned}
$$

Since $\left|{ }^{b} D_{q} f\right|^{p_{1}}$ is convex, we have 


$$
\begin{aligned}
& \int_{0}^{\frac{1}{1+q}} t\left|{ }^{b} D_{q} f(t a+(1-t) b)\right|^{p_{1}} d_{q} t \\
\leq & \int_{0}^{\frac{1}{1+q}} t\left[t\left|b D_{q} f(a)\right|^{p_{1}}+(1-t)\left|{ }^{b} D_{q} f(b)\right|^{p_{1}}\right] d_{q} t \\
= & \frac{1}{(1+q)^{3}\left(1+q+q^{2}\right)}\left|{ }^{b} D_{q} f(a)\right|^{p_{1}}+\frac{q}{(1+q)^{2}\left(1+q+q^{2}\right)}\left|{ }^{b} D_{q} f(b)\right|^{p_{1}}
\end{aligned}
$$

and similarly

$$
\begin{aligned}
& \int_{\frac{1}{1+q}}^{1}\left(\frac{1}{q}-t\right)\left|{ }^{b} D_{q} f(t a+(1-t) b)\right|^{p_{1}} d_{q} t \\
\leq & \frac{2}{(1+q)^{3}\left(1+q+q^{2}\right)}\left|{ }^{b} D_{q} f(a)\right|^{p_{1}}+\frac{-1+q+q^{2}}{(1+q)^{3}\left(1+q+q^{2}\right)}\left|{ }^{b} D_{q} f(b)\right|^{p_{1}} .
\end{aligned}
$$

On the other hand we can easily see that

$$
\int_{0}^{\frac{1}{1+q}} t d_{q} t=\frac{1}{(1+q)^{3}}=\int_{\frac{1}{1+q}}^{1}\left(\frac{1}{q}-t\right) d_{q} t . \text { This completes the proof. }
$$

\section{Conclusions}

In this paper, we establish some Trapezoid and Midpoint type inequalities for $q^{b}$-integrals. In order to validate that their generalized behavior, we show the relation of our results with previously published ones. In the future works, authors can obtain similar inequalities by using the different kind convexities. 


\section{References}

[1] N. Alp, M. Z. Sarikaya, M. Kunt and I. Işcan, "q-Hermite Hadamard inequalities and quantum estimates for midpoint type inequalities via convex and quasi-convex functions", Journal of King Saud University Science, vol. 30, no. 2, pp. 193-203, Apr. 2018, doi: $10.1016 /$ j.jksus.2016.09.007

[2] N. Alp and M. Z. Sarikaya, "Hermite Hadamard's type inequalities for co-ordinated convex functions on quantum integral", Preprint, Dec. 2018. [On line]. Available: https:// bit.ly/2XzUush

[3] S. Bermudo, P. Kórus, and J. Nápoles Valdés, "On q-Hermite-Hadamard inequalities for general convex functions", Acta mathematica hungarica, vol. 162, no. 1, pp. 364-375, Oct. 2020, doi: $10.1007 / \mathrm{s} 10474-020-01025-6$

[4] S. S. Dragomir and C. E. M. Pearce, Selected topics on HermiteHadamard inequalities and applications. Melbourne: RGMIA Monographs, Victoria University, 2000. [On line]. Available: https:/ / bit.ly/ 3nLdEpA

[5] S. S. Dragomir and R. P. Agarwal, "Two inequalities for differentiable mappings and applications to special means of real numbers and to trapezoidal formula", Applied mathematics letters, vol. 11, no. 5, pp. 91-95, Sep. 1998, doi: 10.1016/S0893-9659(98)00086-X

[6] T. Ernst, The history of q-calculus and new method. Uppsala: Uppsala University, 2000. [On line]. Available: https:// bit.ly/2LQkdK5

[7] T. Ernst, A comprehensive treatment of q-calculus. Basel: Birkhäuser, 2012, doi: 10.1007/978-3-0348-0431-8

[8] F. H. Jackson, "On a q-definite integrals", The quarterly journal pure applications mathematics, vol. 41, pp. 193-203, 1910. [On line]. Available: https:// bit.ly/35DhdYy

[9] V. Kac and P. Cheung, Quantum calculus. New York, NY: Springer, 2002, doi: 10.1007/978-1-4613-0071-7

[10] U. S. Kirmaci, "Inequalities for differentiable mappings and applications to special means of real numbers and to midpoint formula", Applied mathematics and computation, vol. 147, no. 1, pp. 137-146, Jan. 2004, doi: 10.1016/S0096-3003(02)00657-4

[11] M. A. Noor, K. I. Noor, and M. U. Awan, "Some quantum estimates for Hermite-Hadamard inequalities", Applications mathematics computation, vol. 251, pp. 675-679, Jan. 2015, doi: 10.1016/ j.amc.2014.11.090 
[12] M. A. Noor, K. I. Noor, and M. U. Awan, "Some quantum integral inequalities via preinvex functions", Applications mathematics computation, vol. 269, pp. 242-251, Oct. 2015, doi: 10.1016/ j.amc.2015.07.078

[13] M. Noor, K. Noor, and M. Awan, "Quantum Ostrowski inequalities for $q$-differentiable convex functions", Journal of mathematical inequalities, vol. 10, no. 4, pp. 1013-1018, 2016, doi: $10.7153 /$ jmi-10-81

[14] J. E. Pěcarić, F. Proschan, and Y. L. Tong, Convex functions, partial orderings and statistical applications. Boston, MA: Academic Press, 1992.

[15] W. Sudsutad, S. K. Ntouyas, and J. Tariboon, "Quantum integral inequalities for convex functions", Journal mathematics inequalities, vol. 9, no. 3, pp. 781-793, 2015, doi: 10.7153/ jmi-09-64

[16] J. Tariboon and S. K. Ntouyas, "Quantum calculus on finite intervals and applications to impulsive difference equations", Advances difference equations, vol. 282, Art ID. 282, Nov. 2013, doi: $10.1186 / 1687-1847-2013-282$

[17] H. Zhuang, W. Liu, J. Park, "Some quantum estimates of HermiteHadmard inequalities for quasi-convex functions", Mathematics, vol. 7, no.2, Art ID. 152, Feb. 2019, doi: 10.3390/ math7020152 